\title{
A case of chronic subdural haemorrhage and its medico-legal implications
}

\author{
Amararatne, $R R G S^{1}$, Jayasundara, $S^{2}$, Vidanapathirana $M^{3^{*}}$
}

\begin{abstract}
Subdural haemorrhage is almost always traumatic and rarely natural. Traumatic Subdural haemorrhage (SDH) occurs following break of the parasagittal bridging vessels due to shearing movement of the head. Clinical features appear in 3 days in acute SDH, in 3 days to 3 weeks in sub-acute SDH and after 3 weeks in chronic SDH. SDHs are aged macroscopically and histopathologically. A 42 years old female knocked down by a bus was admitted to a base hospital. She was unconscious on admission and had nausea, vomiting and headache. By the next day, almost all symptoms were settled except headache and was discharged. Fifteen days later, she developed sudden onset bilateral lower limb weakness and was re-admitted. She was transferred to a tertiary care hospital immediately. The pre-op CT scan showed left frontal chronic subdural hemorrhage (SDH) and the patient was subjected to a burr hole surgery. She was transferred back to the base hospital and according to the medico-legal examination performed 21 days after the incident, the category of hurt was "fatal in the ordinary course of nature". In the remarks column, it was mentioned that it is not clear whether this SDH is due to the fall from the bus or any other incident after that.

This chronic SDH also could be following a separate "new incident" that took place after the traffic accident. Therefore, without dating the chronic SDH accurately, it is not safe to identify the real causative circumstance.
\end{abstract}

Key words: Subdural haemorrhage, aging, histopathology, post-operative samples

\section{Full paper}

\section{Introduction}

Subdural space is a potential space and it is situated between dura and the arachnoid mater. Subdural haemorrhage (SDH) is almost always traumatic and rarely natural, for example in bleeding diathesis. ${ }^{[1]}$ Traumatic SDH occurs following break of the parasagittal bridging vessels due to shearing movement of the head. Clinical features appear in 3 days in acute $\mathrm{SDH}$, in 3 days to 3 weeks in sub-acute SDH and after 3 weeks in chronic SDH. ${ }^{[2]}$ TheSDHs are aged macroscopically and histopathologically. ${ }^{[2]}$

${ }^{1}$ Consultant Judicial Medical Officer, ${ }^{2}$ Medical Officer, Base Hospital, Medico-legal Unit, Base Hospital, Puttalam, ${ }^{3}$ Forensic Medicine, Professor, Department of Forensic Medicine, Faculty of Medical Sciences, University of Sri Jayewardenepura, Sri Lanka.

*Corresponding author: Vidanapathirana M, email: mudithavidana@sjp.ac.lk, Tel: +94772988227

DOI: http://doi.org/10.4038/mljsl.v5i1.734

https://orcid.org/0000-0003-0071-0996
The case under discussion highlights the difficulties encountered in giving a proper medico legalopinionin a case of chronic SDH following a road traffic accident.

\section{Case report}

A 42 years old female knocked down by a bus while walking along a road was admitted to a base hospital. She was unconscious on admission and had nausea, vomiting and headache. However, she did not have injuries on the head and had not lose her memory of the events that took place before the incident. By the next day, almost all symptoms were settled except headache and were discharged.

Fifteen days later, she developed sudden onset bilateral lower limb weakness and was re-admitted. She was transferred to a tertiary care hospital immediately. The pre-op CT scan (Fig. 1) showed left frontal chronic subdural hemorrhage (SDH) and the patient was subjected to a burr hole surgery. Postop CT (Fig. 2) showed a residual thin layer of hypo- 
dense subdural collection with air within and most shift. Further, the brain surface was flattened, hemisphere was not swollen, ventricles were not dilated and herniations were not found.

She was transferred back to the base hospital and according to the medico-legal examination performed likely post-surgical changes with no midline after 21 days of the incident, there were no apparent external head injuries. However, the category of hurt was fatal in the ordinary cause of nature (FIOCN) but it is not clear whether this is due to the fall from the bus or any other incident after that.

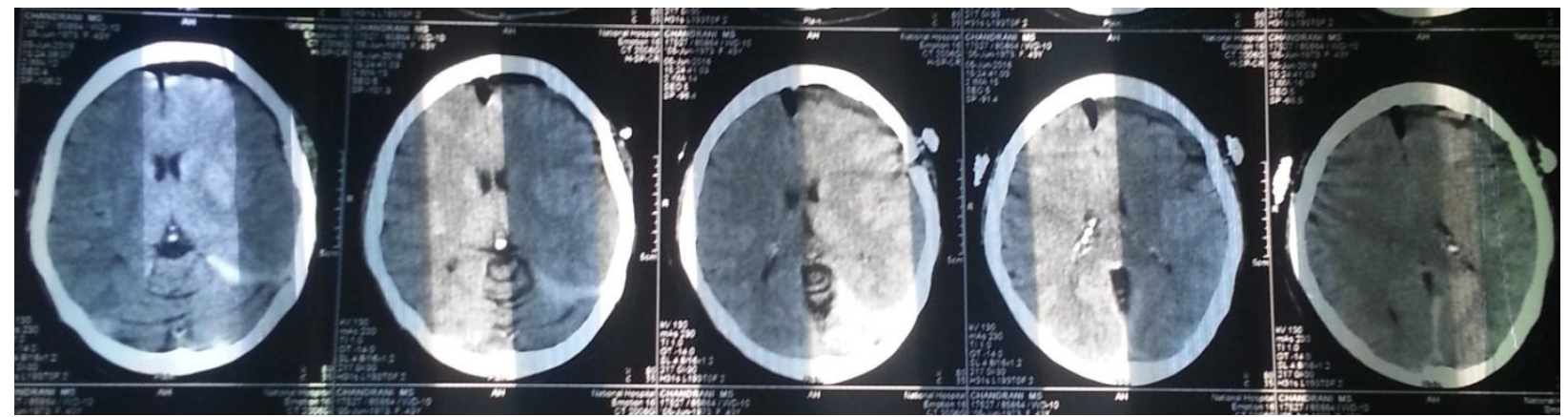

Figure 1: Pre-op CT shows the left frontal chronic SDH

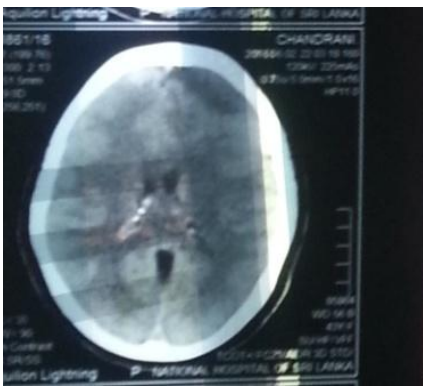

Figure 2: Post-op CT scan

\section{Discussion}

In this case, the aging of the SDH is very important for the establishment of the cause-effect relationship and then the accurate identification of the circumstance. She developed clinical features such as unconsciousness, nausea, vomiting and headache immediately after the head injury. In acute SDHs, the clinical features usually appear within 3 days. ${ }^{[2]}$ If patient survives, the SDH gets organized. Initially the outer membrane is formed and in about 2 weeks an inner membrane is formed. These layers are initially yellow in colour due to hemosiderin laden macrophages. The central blood clot is organized by developing granulation tissue with new blood vessel formation. In one month, usually, no central clot is found and the inner and outer membranes adhere. Now the membranes are transparent, colourless or slightly yellow and the punctate deposits represent haemosiderin laden histiocytes. ${ }^{[1,2]}$ However, in this case, the surgically removed SDH was not available for histopathological studies.

In this case, the patient developed sudden onset bilateral lower limb weakness and the CT scan taken

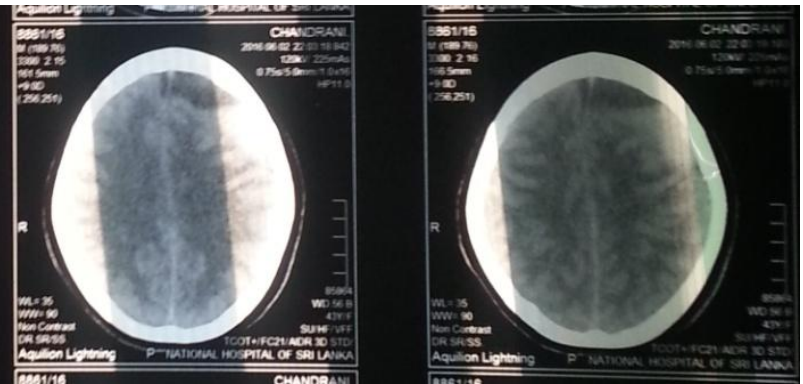

after 15 days of the accident showed chronic SDH. In Chronic SDHs, the clinical features appear usually after about 21 days. $^{[2]}$ Further, by this time the existing organized SDH should have inner and outer membranes. It usually appears in elderly people, chronic alcoholics, epileptic patients, with ventriculoperitoneal shunts and with coagulopathy. ${ }^{[2]}$ Identification of risk factors of chronic SDH can offer clinical follow-up strategies for patients after episodes of head injury. A nationwide populationbased study in Taiwan, showed that old age $(\geq 75$ years), male gender, and coexisting hydrocephalus are significantly predictive factors, irrespective to their medical co morbidities. ${ }^{[3]}$ However, in this case, the victim was a 42 year old adult female with no significant past medical or surgical history.

After 15 days of the incident, she developed sudden onset bilateral lower limb weakness. With time, the acute SDH gets organised. However, it can produce clinical features as in this case due to sudden enlargement of the SDH following re-bleeding or absorption of tissue fluid into the concentrated central liquid of the SDH. ${ }^{[2]}$ Sometimes, SDH can also be enlarged due to formation of subdural hygromas due 
to absorption of the CSF in to the SDH. ${ }^{[4]}$ However, the diagnosis of re-bleeding in a chronic SDH should only be suspected if characteristic autopsy and microscopic findings are observed. ${ }^{[2]}$ Further, in this case, except for the road traffic accident, no previous or subsequent history of head injury was available. However, in 50\% chronic SDH patients, no history of head injury is available because re-bleeding can occur due to trivial head injuries. ${ }^{[5]}$

Further, for the identification of the SDHs, autopsy examination is more sensitive than CT scans and the CT scans should not be used to estimate the age of $\mathrm{SDH} .{ }^{[2]}$ MRI can detect SDHs of different ages, but should not also be used to estimate the age of the injury. ${ }^{[2]}$ Similarly, in this case, the radiologist had not mentioned about membranes or central clot and the accurate aging cannot be ascertained based on the CT scan findings.

Chronic SDH occurs due to subsequent minor trauma causing new bleeding from new blood vessels of granulation tissue and therefore, prevent the fusion of these membranes. Sometimes, re-bleeding can rupture the inner membrane and form a second SDH and a second inner membrane. Therefore, multiple neo-membranes can be developed by re-bleeds. If subsequent minor trauma damages the underlying arachnoid mater, the CSF can leak in to the subdural space and can cause a subdural hygroma. ${ }^{[2]}$ Further, in CT scans of chronic SDHs, mixed densities may results in multiple episodes of trauma. ${ }^{[6]}$

Under the chronic SDH, usually, the brain surface is flattened, midline is not shifted, hemisphere is not swollen, ventricles are not dilated and herniations are not found. In this case, the post-op CT scan showed similar findings. ${ }^{[2]}$ Absence of herniations and infarctions indicate that the chronic SDH accumulates slowly and has not increased the intracranial pressure.

Though she lost her consciousness immediately after the incident, the absence of retrograde amnaesia The category of hurt was ascertained as Fatal in the ordinary course of nature (FIOCN) but it was further mentioned that it is not clear whether this $\mathrm{SDH}$ is due to the fall from the bus or any other incident after that. This SDH could be following a new trivial incident that took place before or after the traffic accident. Such events that occur after the incident are called "Novus actus interveniens", that is the involvement of a new act destroying the chain of events. $^{[7]}$

\section{Conclusion}

Therefore, without accurate dating of the chronic $\mathrm{SDH}$, it is not safe to identify the real causative circumstance. This highlights the importance of submitting the surgically resected tissues for further investigations such as histopathology especially in medico-legal cases.

\section{References}

1. Knight B, Forensic Pathology, $2^{\text {nd }}$ ed, London: Arnold;1996

2. Spitz WU, Spitz DJ, Spitz and Fisher's Medicolegal investigation of death, $4^{\text {th }}$ ed, USA: Charles C Thomas; 2006

3. Tseng JH, Tseng MY, Liu AJ, Lin WH, Hu HY, Hsiao SH. Risk factors for chronic subdural hematomaafter a minor head injury in the elderly: a population-basedstudy. Biomed Res Int 2014;2014:218646.

4. Wang Y, Wang C, Liu Y. Chronic subdural haematoma evolving from traumatic subdural hydroma. Brain Inj. 2015;29(4):462-5.

5. Lee HC, Chong S, Lee JY, Cheon JE, Phi JH, Kim SK, Kim IO, Wang KC. Benign extracerebral fluid collection complicated by subdural hematoma and fluid collection: clinical characteristics and management. Childs Nerv Syst. 2017 Sep 9. doi: 10.1007/s00381-017-3583y.

6. Park HR, Lee KS, Shim JJ, Yoon SM, Bae HG, Doh JW. MultipleDensities of the Chronic Subdural Hematoma in CT Scans. J Korean NeurosurgSoc 2013 Jul;54(1):38-41.

7. Osborne PH. The Law of Torts, Toronto : Irwin Law; 2015. 